\title{
Therapeutic Options for the Treatment of Pulmonary Hypertension
}

\author{
Rownak Jahan Tamanna ${ }^{1}$ \\ ${ }^{1}$ Department of Cardiology, BIRDEM \\ Address for Correspondance \\ Dr. Rownak Jahan Tamanna, Department of Cardiology, BIRDEM, Dhaka \\ e- mail : rownakjt@yahoo.co.in
}

\begin{abstract}
The therapy of pulmonary hypertension depends on the identification of underlying contributing factors. pulmonary arterial hypertension (PAH), which can be idiopathic or related to connective tissue disease, portal hypertension, HIV disease, ingestion of certain drugs or toxins, or congenital heart disease, had no specific therapy until recently. However, the past decade has seen remarkable progress, and these heretofore devastating and usually lethal forms of pulmonary hypertension now often respond to one form of therapy or another, leading to improved functional capacity and even survival. The following will consider the major pharmacotherapy's now available for PAH and suggest a framework for therapeutic decision-making.
\end{abstract}

Key word : Pulmonary hypertension

\section{Introduction}

Pulmonary hypertension refers to an abnormal elevation of pulmonary artery (PA) pressure (mean PA pressure $>25 \mathrm{~mm}$ $\mathrm{Hg}$ at rest and $30 \mathrm{~mm} \mathrm{Hg}$ with exercise).

It is a rare disease of unknown etiology, whereas secondary pulmonary hypertension is a complication of pulmonary, cardiac and extrathoracic conditions. Unrelieved pulmonary hypertension can lead to right heart failure. The diagnosis should be suspected in patients with increasing dyspnoea on exertion and a known cause of pulmonary hypertension. Two-dimensional echocardiography with Doppler flow studies is the most useful imaging modality in patients with suspected pulmonary hypertension. Treatment options are diverse. Lung transplantation remains an option for selected patients with pulmonary hypertension that does not respond to medical management.

\section{Treatment Options for Pulmonary Hypertension}

General Approach

Some therapies have long been available to treat pulmonary hypertension. Adequate diuresis brings about substantial symptomatic improvement. Oxygen should be provided to hypoxemic patients. Digoxin is administered for supraventricular arrhythmias or right ventricular dilatation. Warfarin anticoagulation is routinely administered to patients with moderate to severe PAH, aiming for an INR between 1.5 and 2.5. Phlebotomy has been recommended when the hematocrit exceeds 56, particularly in patients with congenital heart disease. Calcium channel blockers, sometimes in high doses, were considered the most effective pharmacotherapy's for pulmonary hypertension prior to the advent of newer specific therapies. ${ }^{1}$ With the exception of anticoagulation and calcium channel blocker therapy in responders, these therapies should be considered mainly palliative and although they can be quite useful for ameliorating symptoms, they do not significantly affect the natural history of the disease.

Newer and more effective pharmacotherapy's for PAH are now available, starting with prostacyclins in 1996 and, more recently, endothelin receptor antagonists and phosphodiesterase 5 inhibitors. Relative merits of the different therapies, suggested applications, possible combinations, and newer therapies are discussed below.

Prostacyclins

Epoprostenol- The therapeutic administration of prostacyclin (epoprostenol) as a continuous intravenous infusion to patients with idiopathic PAH was first reported during the 1980 s. $^{2}$ and a randomized, controlled trial on patients with idiopathic PAH was reported in $1996 .{ }^{3}$ Enrolling 81 patients, this trial demonstrated a significant improvement in 6minute walk distance, the major outcome variable, as well as in pulmonary hemodynamics, oxygenation and survival. Based on these the US Food and Drug Administration (FDA) approved epoprostenol as a continuous intravenous therapy for patients with class III or IV idiopathic PAH. It is still considered the therapy of first choice for patients in the NYHA class IV and the last resort when other therapies have failed.

Despite its demonstrated efficacy, intravenous epoprostenol is far from an ideal therapy. It requires daily mixing and must be kept in a cold pack to avoid degradation at room temperature. The infusion pump and cold pack can be carried in a fanny pack, but are cumbersome for many patients. In addition, the need for a permanent transcutaneous intravenous catheter poses risks for line infection and sepsis and sudden occlusion of the catheter can precipitate hemodynamic collapse because of the several minute half-life of the drug. 
Treprostinil. The shortcomings of intravenous epoprostenol have stimulated the search for alternative therapies. Treprostinil, a prostacyclin analog with a half-life of several hours, was approved by the FDA for subcutaneous administration in 2002. Stable at room temperature, it requires no cold packs and can be administered using a continuous infusion pump that approximates the size of a pager. The drug was shown to be safe and effective in a pivotal trial that demonstrated an average improvement in 6-minute walk distance of 16 meters. ${ }^{4}$ Unfortunately, many patients encounter pain at the infusion site, and a significant minority of patients finds the drug intolerable. In late 2004, the FDA approved intravenous treprostinil to treat class II to IV PAH. An inhaled form of treprostinil is currently under investigation, and preliminary reports suggest that the hemodynamic effect is sustained for more than 2 hours after only a few inhalations. ${ }^{5}$

Iloprost. Iloprost, another prostacyclin analog with a halflife between those of epoprostenol and treprostinil, received FDA approval only during 2004. A randomized controlled trial in patients with PAH or chronic thromboembolic pulmonary hypertension showed a significant improvement in the 6 -minute walk distance, ${ }^{6}$ but $5 \%$ of patients experienced syncope, presumably because they exerted excessively after the drug effect had waned. The duration of hemodynamic effect averages approximately 90 minutes after inhalation, and the drug requires 6 to 9 nebulizer treatments per 24 hours, each treatment requiring up to 10 to 20 minutes, many patients find the frequency of treatments cumbersome.

Oral Beraprost. Beraprost, another prostacyclin analog with a longer half-life than epoprostenol, is suitable for oral administration. Uncontrolled trials in Japan, where the drug is currently available, have suggested beneficial actions in patients with pulmonary hypertension related to congenital heart disease as well as in chronic thromboembolic pulmonary hypertension. ${ }^{7}$ But a 12 month randomized controlled trial in the United States failed to show sustained benefit, even though there was significant improvement in the 6-minute walk distance at the 3 and 6 month time points. ${ }^{8}$ For this reason, the FDA will not approve oral prostacyclin therapy in the United States at the present time. However, other oral therapies (treprostinil) are under investigation.

\section{Endothelin Receptor Antagonists}

A number of endothelin receptor blockers have been developed to treat PAH that vary in their specificity for receptor blockade.

Nonselective Endothelin Receptor Blockade. Presently, only one endothelin receptor antagonist has received FDA approval in the United States -- the oral nonselective blocker, bosentan. This drug was studied in an earlier randomized controlled trial that demonstrated not only a significant improvement in 6-minute walk distance, but also improvements in pulmonary hemodynamics. A subsequent 16-week pivotal randomized controlled trial (BREATHE-1) confirmed the improvement in 6-minute walk distance (44 meters greater compared with placebo) and also showed significant improvements in NYHA functional class and quality-of-life scores, as well as a significant decrease in the rate of clinical worsening. Since its FDA approval in 2001, bosentan has been the most widely prescribed specific pulmonary hypertension therapy. Subsequent long-term follow-up studies demonstrate a 2 -year survival of $89 \%$ in patients begun on bosentan, with $70 \%$ remaining on monotherapy. ${ }^{9}$

The drug is usually well tolerated, but can exacerbate fluid retention and may not bring about clinical improvements for as long as 2 to 3 months after initiation. For this reason, even though the drug was approved for therapy of both class III and class IV patients with idiopathic PAH or PAH related to connective tissue disease, routine monotherapy for class IV patients is inadvisable. Also, the drug causes greater than a 3 -fold elevation in liver transaminases in approximately $10 \%$ of patients, and liver function tests must be monitored monthly for the duration of therapy. The recommendation is to start at $62.5 \mathrm{mg}$ twice daily for a month and then advance to $125 \mathrm{mg}$ twice daily if liver function tests are acceptable.

Specific A-receptor Blockers. Two specific A-receptor blockers are currently under investigation for the therapy of PAH. The first, sitaxsentan, is approximately 6000-fold more specific for the A than B receptor and has a longer half-life that permits once-daily oral administration. Tested in a 12week randomized controlled trial (STRIDE-1), it failed to show a statistically significant benefit in the major outcome variable, maximal oxygen uptake at the $100 \mathrm{mg}$ per day dose, significant improvements in the 6-minute walk distance, pulmonary hemodynamics, and NYHA classification. ${ }^{10}$ Because of the failure to show significant benefit in the major end point, further trials have been performed including STRIDE-2, in which daily doses of $50 \mathrm{mg}$ and 100 mg were compared with placebo and open-label bosentan at $125 \mathrm{mg}$ twice daily. A preliminary report shows that 100-mg daily dose of sitaxsentan improves 6-minute walk distance and NYHA class compared with placebo and comparably to bosentan, but the 50-mg daily dose failed to show significant benefit. ${ }^{11}$ Liver toxicity tended to be less at the $100-\mathrm{mg}$ dose of sitaxsentan compared with bosentan $125 \mathrm{mg}$ twice daily (3\% vs $11 \%)$, Sitaxsentan offers the advantages of once daily administration and possibly less liver toxicity than bosentan, but efficacy appears to be comparable. It is currently under consideration for approval by the FDA. 
Ambrisentan, another endothelin receptor antagonist that is 18 times more specific for the A than B receptor, is currently undergoing investigation. Like sitaxsentan, it is a once-aday oral therapy, and preliminary findings suggest that it poses a low risk of liver toxicity.

\section{Phosphodiesterase 5 Inhibitors}

Phosphodiesterase-5 metabolizes cyclic GMP, decreasing intracellular levels, and limiting vasodilator effects. Phosphodiesterase-5 inhibitors slow the metabolism of cyclic GMP, intensifying the vasodilator actions. Presently, only one phosphodiesterase inhibitor, sildenafil, has been tested in PAH and was recently approved by the FDA for the therapy of PAH patients at a dose of $20 \mathrm{mg} 3$ times daily. A pivotal randomized controlled trial of sildenafil (SUPER-1) showed statistically significant improvements in 6-minute walk distance (45-50 meters), pulmonary hemodynamics and NYHA class over 12 weeks. Headache and epistaxis were the only side effects encountered more often than with placebo. Favorable effects are often seen within days of drug initiation. A trial to test the efficacy of a longer-acting phosphodiesterase-5 inhibitor, tadalafil, is just being started. This would presumably allow once-daily or even less frequent administration.

\section{New Potential Therapies}

A number of other therapeutic approaches to PAH are being tested or contemplated The thromboxane A-2 antagonist, terbogrel, was studied in a randomized controlled trial that was stopped prematurely when many patients in the treatment arm experienced leg pain S Statins are under consideration as a pulmonary hypertension therapy because they possess anti-inflammatory and antiproliferative actions and have been effective in rodent models of PAH. ${ }^{12}$ Estrogens have been shown to blunt pulmonary hypertension in some animal models. ${ }^{13}$ Serotonin receptor inhibitors are being considered as pulmonary hypertension therapies. Gene therapy approaches are also under consideration, such as transfection with the nitric oxide or prostacyclin synthase genes, but suitable long-acting vectors have not yet been developed.

\section{Combination Therapy}

The concept of combining agents with fundamentally different mechanisms of action is attractive. A study demonstrated that the addition of sildenafil in patients who deteriorated while on iloprost therapy alone restored clinical stability, associated with improvements in 6-minute walk distance. ${ }^{14}$ In the randomized controlled BREATHE-2 trial, bosentan combined with intravenous epoprostenol showed trends toward improved pulmonary hemodynamics compared with epoprostenol with placebo. ${ }^{15}$

Preliminary results were recently released on the STEP-2 trial that randomly added inhaled iloprost vs placebo in patients already receiving bosentan therapy. A significant improvement in 6-minute walk distance was attributable to the addition of iloprost when administered an average of 6 times daily. ${ }^{16}$ Few data are available on the combination of endothelin receptor antagonists and sildenafil, but a recent case series described improvement in functional status (> 100-m improvement in 6-minute walk distance) after sildenafil was added in 9 patients deteriorating after a year on bosentan monotherapy. 17

Some patients, anecdotally, are being treated with all 3 FDA approved PAH therapies, along with statins and traditional therapies. However, this is very expensive, and we cannot be certain, in the absence of data, that these combinations are beneficial. A number of randomized controlled trials are being initiated that should provide answers to many of the questions surrounding combination therapy over the next several years.

\section{Surgical Therapeutic Options}

Lung and heart/lung transplantation as well as atrial septostomy have been available for years to treat severe PAH. With the advent of effective pharmacotherapy, however, the need for these interventions has diminished, but they are still considerations for patients who fail to respond favorably and remain in class III or IV after at least several months of optimal pharmacotherapy.

\section{Conclusions}

Although we have long had a variety of palliative treatments for pulmonary hypertension that should not be forgotten, in recent years 3 new pharmacotherapeutic categories have been added to our therapeutic regimen for PAH: the prostacyclins, endothelin receptor antagonists and phosphodiesterase-5 inhibitors. Each year, new agents are being added to each of these new categories, and newer therapeutic avenues are being investigated or contemplated. However, many questions remain unanswered, such as whether some patients should be initiated on combination therapy, what to do when patients fail to respond adequately to initial therapy, and when to use inhaled therapies. Because of the complexity of managing PAH patients, referral to centers specializing in pulmonary hypertension is recommended, partly to give them the opportunity to participate in clinical trials. 


\section{References}

1. Rich S, Kaufmann E, Levy PS. The effect of high doses of calcium-channel blockers on survival in primary pulmonary hypertension. N Engl J Med. 1992;327:76-81

2. Olschewski H, Rose F, Schermuly R, et al. Prostacyclin and its analogues in the treatment of pulmonary hypertension. Pharmacol Ther. 2004;102:139-53

3. Barst RJ, Rubin LJ, Long WA, et al. A comparison of continuous intravenous epoprostenol (prostacyclin) with conventional therapy for primary pulmonary hypertension. $N$ Engl $J$ Med. 1996;334:296-301

4. Simonneau G, Barst RJ, Galie N, et al;Treprostinil Study Group. Continuous subcutaneous infusion of treprostinil, a prostacyclin analogue, in patients with pulmonary arterial hypertension: a double-blind, randomized, placebo-controlled trial. Am J Respir Crit Care Med. 2002;165:800-04

5. Voswinckel R, Enke B, Kreckel A, et al. Pharmacological testing with inhaled treprostinil for pulmonary hypertension and 9 month treatment experience. Proc Am Thorac Soc. 2005;2:A199

6. Olschewski H, Simonneau G, Galie N, et al; Aerosolized Iloprost Randomized Study Group. Inhaled iloprost for severe pulmonary hypertension. $N$ Engl J Med. 2002; 347:322-29

7. Suzuki H, Sato S, Tanabe S, Hayasaka K. Beraprost sodium for pulmonary hypertension with congenital heart disease. Pediatr Int. 2002;44:528-29

8. Barst RJ, McGoon M, McLaughlin V, et al; Beraprost Study Group. Beraprost therapy for pulmonary arterial hypertension. J Am Coll Cardiol. 2003;41:2119-25
9. Rubin LJ, Badesch DB, Barst RJ, et al. Bosentan therapy for pulmonary arterial hypertension. $N$ Engl J Med. 2002; 346:896-903

10. Barst RJ, Langleben D, Frost A, et al; STRIDE-1 Study Group. Sitaxsentan therapy for pulmonary arterial hypertension. Am J Respir Crit Care Med. 2004;169:441-47

11. Barst RJ, Langleben D, Badesch D, et al. The STRIDE-2 trial: does selectivity matter in endothelin antagonism for PAH? Proc Am Thorac Soc. 2005:2:A300

12. Kao PN. Simvastatin treatment of pulmonary hypertension: an observational case series. Chest. 2005;127:1446-52

13. Resta TC, Kanagy NL, Walker BR. Estradiol-induced attenuation of pulmonary hypertension is not associated with altered eNOS expression. Am J Physiol Lung Cell Mol Physiol. 2001;280:L88-97

14. 23.Ghofrani HA, Rose F, Schermuly RT, et al. Oral sildenafil as long-term adjunct therapy to inhaled iloprost in severe pulmonary arterial hypertension. J Am Coll Cardiol. 2003; 42:158-64

15. Humbert M, Barst RJ, Robbins IM, et al. Combination of bosentan with epoprostenol in pulmonary arterial hypertension: BREATHE-2 .Eur Respir J. 2004;24:353-59

16. Olschewski H, Simonneau G, Galie N, et al; Aerosolized Iloprost Randomized Study Group. Inhaled iloprost for severe pulmonary hypertension. $N$ Engl J Med. 2002; $347: 322-29$

17. Badesch DB, Abman SH, Ahearn GS, et al; American College of Chest Physicians. Medical therapy for pulmonary arterial hypertension: ACCP evidence-based clinical practice guidelines. Chest. 2004;126 (1 suppl):35S-62S 